\title{
Finite Element Analysis of Glass Fiber-Reinforced Polyamide Engine Oil Pan Subjected to Localized Low Velocity Impact from Flying Projectiles
}

\author{
Zakaria Mouti ${ }^{1}$, Keith Westwood ${ }^{2}$, Darren Long ${ }^{2}$ and James Njuguna ${ }^{1^{*}}$ \\ 1 Centre for Automotive Technology, Department of Sustainable Systems, Cranfield University, \\ Bedfordshire, MK43 0AL, UK, z.mouti@cranfield.ac.uk \\ ${ }^{2}$ Eaton, Automotive Group, West Midlands, DY5 2LB, UK, keithwestwood@eaton.com \\ * Author to whom correspondence should be addressed: \\ j.njuguna@cranfield.ac.uk, Tel.: +44-1234-75-4186, Fax: +44-1234-75-1671
}

\begin{abstract}
This paper investigates low velocity impact involving a glass fiber-reinforced polyamide engine oil pan as part of a complete new development of thermoplastic components. The assessment of the impact resistance has driven the need to employ LS DYNA for finite element modeling in order to benchmark and predict the strength and fracture behavior of stressed plastic parts.

In order to develop a reliable predictive capability and to validate simulations, complete components were manufactured by injection molding techniques for the experimental samples. Low velocity impact investigations were carried out using a gas gun and a falling weight tester in order to simulate impact events to which the oil pan is subjected whilst in operational service. This was intended to point out damage tolerance and failure mechanisms likely to occur in the structure.

The study results show the significant contribution of the design in terms of shock absorption. Specific oil pan design with protective ribbing combined with a superior material considerably improves the impact resistance. The paper provides results and discussions on experimental and finite element analysis investigations before concluding with some remarks.
\end{abstract}

\section{Keywords}

Finite element analysis, low velocity impact, impact resistance, glass fiber reinforced polyamide, thermoplastics, oil pan 


\section{Introduction}

There are many studies that have been performed in order to investigate the impact properties of thermoplastic composites. However there is little knowledge for their response to localized low velocity impact. Low velocity impacts are known to induce damage to the composite in the form of matrix cracking, delamination, debonding and fiber breakage [1-10]. A number of studies on the low velocity impact performance of thermoplastic-matrix composites have been conducted but, in most cases, the composites were fully laminated into relatively rigid plates made of polyethylene terephthalate $[2,6]$, polypropylene [4,8,9], polyethylene [10-13] and PEEK [14,15] fiber reinforcement.

Research has shown that composites are capable of absorbing energy and dissipating it by various fracture and elastic processes when subjected to a low velocity impact [8]. The ability of these materials to absorb energy elastically depends on the mechanical properties of the matrix and fibers, the interfacial strength, the velocity of impact and the size of the component. Polymer matrix composites are known to be highly susceptible to internal damage caused by transverse loads even under low velocity impact [9]. For the effective use of polymer matrix composites in higher performance applications, it is important to understand the cause of damage formation under low velocity impact conditions as well as the potential for improvement of damage resistance characteristics of composites.

Concerns of this paper are the components located underneath the engine and in particular the engine oil pan. Classically made of stamped steel or cast aluminum, a new way is explored to manufacture oil pans with polyamides. As one of the lowest parts in a vehicle, the potential risk of impacts from flying debris may affect the component integrity, like typically a stone collected from the road and projected into the oil pan. Its design is made in such a way that the connection to the engine is consistent with the metal construction previously used. A noticeable facet of this new design is the ribbing which gives a dimensional stability to the structure and also helps to keep the main structure safe after an impact by dissipating the impact energy. The impact resistance of a component is not only influenced by the material, the manufacturing processes and the external conditions but also by the structural design employed.

A growing interest is to employ numerical methods to assess the impact loading of polymer materials $[16,17]$. Yet, the computational modeling of these materials faces up to difficulties. Short glass fibers reinforced polyamides have a non linear behavior under mechanical loading that is not easily reproducible by conventional material models with roots in metals theory $[18,19]$.

An ideal material model for thermoplastics ought to be able to manage large range of temperatures, the dependency of stress-strain relation on stress level, the rate-dependency, the deformation-induced anisotropy and also other frequent observed characteristics $[18,19]$. Material tests are included in this 
paper providing relevant information and are also needed for calibrating the material model. Then, the model is validated by numerical simulations of the component and checked with experimental observations.

\section{Experiments}

\section{Materials and test samples manufacture}

The material in question is the Ultramid ${ }^{\circledR}$ A3HG7 manufactured by BASF. It is an easy flowing and lowmelt viscosity polyamide 66 reinforced with $35 \mathrm{wt} \%$ of short glass fiber. It is heat stabilized and has enhanced resistance to heat aging. The glass fibers have an average length (I) of $300 \mu \mathrm{m}$ and diameter (d) of $13 \mu \mathrm{m}$, thus yielding an average aspect ratio (I/d) of 23 . The material is initially extrusion compounded into pellets by dry blending glass fibers with PA66 pellets and then are injection molded into test samples.

The tensile samples were injection molded into a dumbbell shape to conform to standard ISO 527 following the dimensions of test specimen type $1 \mathrm{~A}$. The flexural samples were injection molded to conform to standard ISO 178. The oil pan samples were injection molded into the required shape with a single impression mold and feeding gate. The process cycle consists of four stages: clamping, injection, cooling and ejection. Typically, prior to the injection phase, the clamping unit pushes two mold halves together to keep the mold closed while the material is injected. The raw plastic is melted by heat and pressure (around $280^{\circ} \mathrm{C}$ and 100 bars), and advances towards the mold for fast injection while the buildup of pressure packs and holds the material. The molten plastic inside the mold begins to cool as soon as it makes contact with the interior mold surfaces into the desired shape. The packing of material allows additional material to flow into the mold to reduce the amount of visible shrinkage. After the required cooling time, the material solidifies and the part is ejected from the mold.

\section{Quasi-static tests}

An Instron 5500 Universal Testing Machine with Merlin application software and utilizing a National Instruments tool box was used which were configured for data acquisition. The Instron 5500 test rig has a screw driven cross-head which is ideal for incremental displacements. Each test was performed five times therefore five samples per condition were used to generate accurate captures of the material behavior at room temperature of $20^{\circ} \mathrm{C}$. 


\section{Low velocity impact tests}

Low velocity impact tests were conducted using an instrumented falling weight impact device (see Figure 1). The device was equipped with data acquisition system to acquire force versus time data. Using this machine, impact energy and velocity can be varied by changing the mass and height of the dropping weight. The velocity of the falling drop mass is measured just before it strikes the specimen. It is also fitted with pneumatic rebound brake, which prevents multiple impacts on the specimen.

An air gas gun (see Figure 1) and $10 \mathrm{~mm}$ projectile with a cylindrical beveled front end and a badminton ball profile to control the flight stability was also utilized in this study. The velocity of the projectile is determined by the amount of pressure set and stored in a reservoir prior the shot. The range of velocities attained varied from 8 to $70 \mathrm{~m} / \mathrm{s}$ and were determined just prior to impact using optical sensors. A speed camera records the impact event. A laser is mounted at the back of the barrel permitting the alignment of the projectile to point towards the selected target spot. The impact distance is kept constant to allow reproducibility under identical impact conditions. The gun is mounted on a sliding support to run the gun to the exact position of shooting.
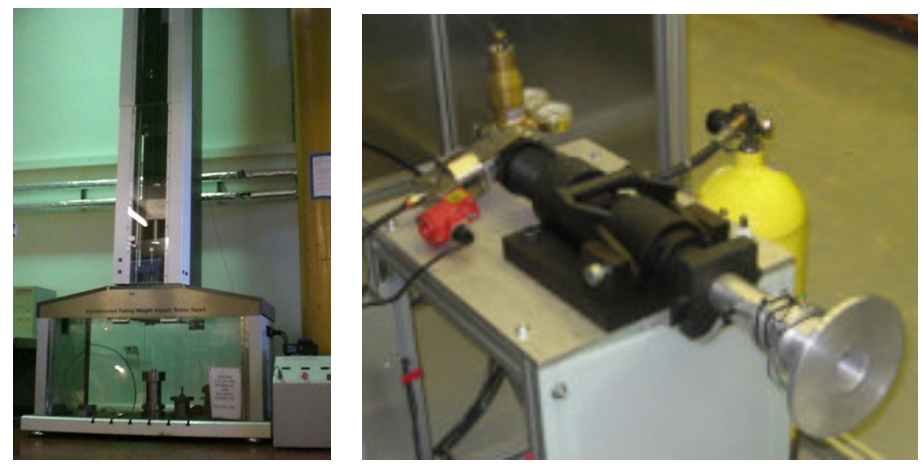

Figure 1: Falling weight impact device (left) and air gas gun (right)

Every result that leads to a possible leak or a break in the base wall structure is to be avoided. The damage assessment is mainly realized by visual inspection. Separate investigations are underway investigating the consequences of micro-fractures. Impact testers were used to simulate the loading conditions to which the composite component is subjected whilst in operational service and hence failure modes and mechanisms likely to occur are reproduced. Oil pan samples were tested under low velocity impacts with impact energies varying from 3 to 12 joules depending on the exposed areas that the oil pan is likely to face from road stones during normal road operations. The oil pan is impacted empty without oil at room temperature of $20^{\circ} \mathrm{C}$. It has a unique design and material composition with no welded parts on it. All impacts are perpendicular to target surfaces which have more critical effects than an angular impact that can rebound without having completely transferred its energy to the target. 
The oil pan samples are constrained, bolted onto a metallic plate and held down on a support. Only a single impact scenario is considered and repeated impacts scenario will be envisaged for further tests.

\section{Modeling and simulation}

Tensile and flexural tests were simulated using respective standards ISO 527-1A and ISO 178. Models were meshed in 2D shell elements as the final product. The experimental test results were used to calibrate the model in order to obtain reliable numerical predictions. The tensile and flexural tests were used to calibrate the model and the falling weight and gas gun impact tests were used to validate the model.

In the impact tests involving projectile or impactor with the oil pan model, an automatic surface to surface contact algorithm is formulated to prevent interpenetration. This contact is non-oriented meaning that it can detect penetration from either side of a shell element. The experimental results were used to determine the parameters to input for each model. The simulations are based on the stress-strain relation with fixed conditions to describe a specific situation at each time. For simplicity, the short glass fiber reinforced polyamide material is assumed to be isotropic and rate independent therefore the visco-elastic effects is ignored. The Von Mises yield condition and isochoric associative plastic flow is assumed [19]. The material model is based on LS-DYNA Piecewise Linear Plasticity. The stress-strain curve in the elastic region is modeled as rate independent up to a determined yield point where deformations are recoverable. Then beyond that point, the curve is decomposed into an elasticplastic model where plastic deformations become significant. The material failure was considered using strain failure criterion, strain corresponding to stress at break. If the calculated effective plastic strain for any element exceeds the predefined value, the element is removed from the model and the simulation continues with the eroded model. However, there is a drawback with plastics, if the strain rate increases the failure strain drops but the model is built on knowledge that this parameter is constant and independent. At each specific condition, a related stress-strain relation is loaded to avoid the strain rate variation.

\section{Results and discussions}

\section{Quasi-static tests results}

Tensile tests on PA66-GF35 were performed to assess its mechanical properties and also to collect relevant data for the simulations. Figure 2 shows the tensile load versus the extension for PA66-GF35. 
Figure 3 shows the stress-strain relation of PA66-GF35 in tension stress condition. This relation is calculated from the tensile load and extension of Figure 2. Each curve represents the average behavior observed made from 10 tests. PA66-GF35 tensile samples undergone a maximum traction force of $7 \mathrm{kN}$ and extended by $5.2 \mathrm{~mm}$. The transition between elastic to plastic domain is noticeable and occurs at around $2 \mathrm{kN}$ below $1 \mathrm{~mm}$ extension. The simulated stress-strain relation is done following the experiments in where only the effective plastic stress-strain relation is implemented into the model. The elastic domain is defined by the Young's modulus up to the set yield point arbitrary chosen since there is no clear transition between the elastic and the plastic domain with thermoplastics. The simulated behavior is slightly under-predicted in the elastic domain. This is due by the difficulty to determine or define the yield transition in these materials. However, the results obtained after the calibration of the model correlate the findings in the experiments.

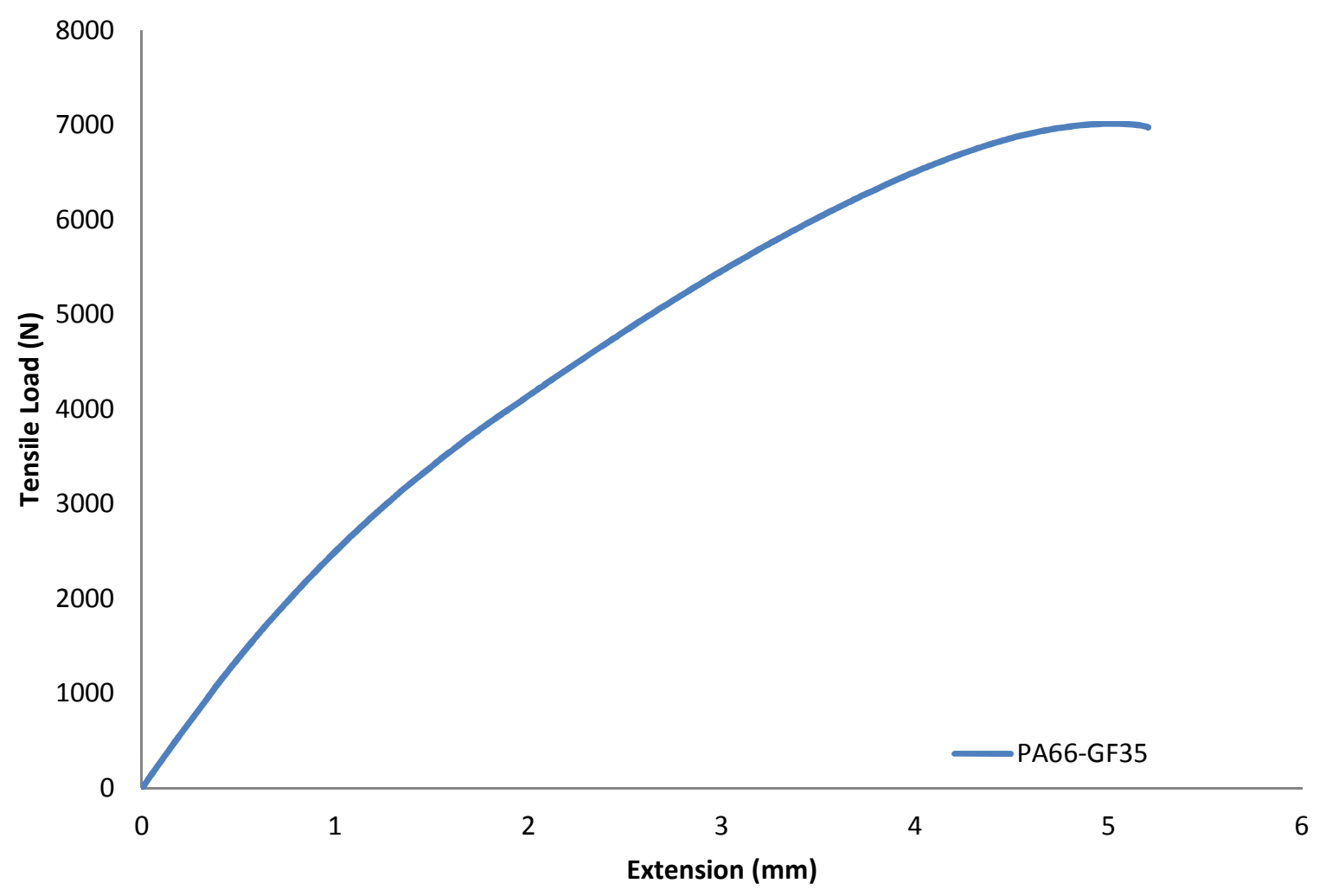

Figure 2: Tensile load / extension relation performed at $1 \mathrm{~mm} / \mathrm{min}$ 


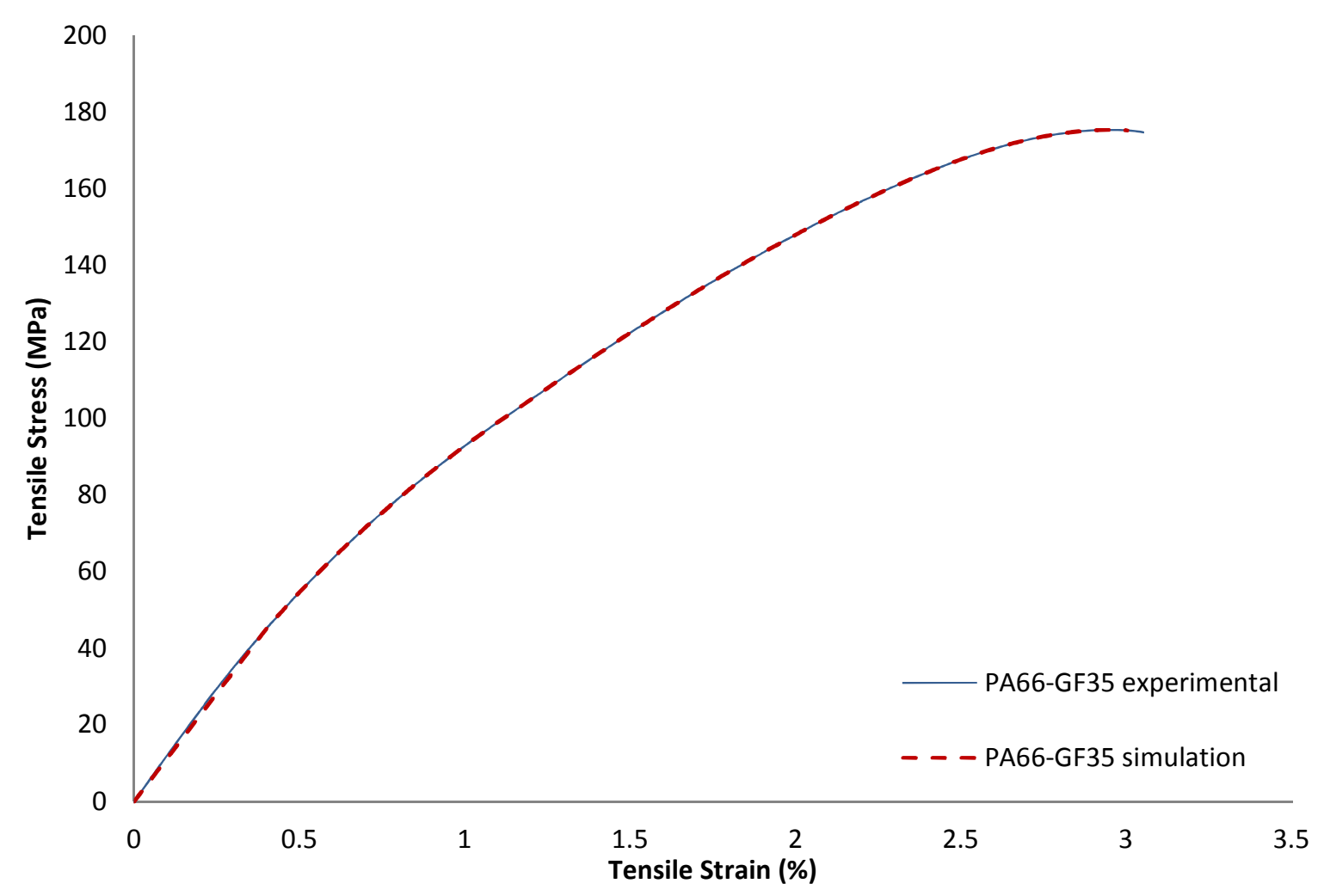

Figure 3: Experimental and calibrated simulation of the tensile stress / tensile strain relation

\section{Low velocity impact test results}

In a falling weight test, the incident velocity of the impactor can be determined from the equations of motion or by using optical sensors located just above the target. The impactor is instrumented, enabling the force versus time characteristics to be determined and also contain a displacement transducer in order to permit the determination of energy dissipation during the impact event. A wide range of geometries can be tested, thereby enabling more complex components to be tested. As it is dependent on the sample configuration and the preparation methods, it is used for comparing and ranking materials and designs. 


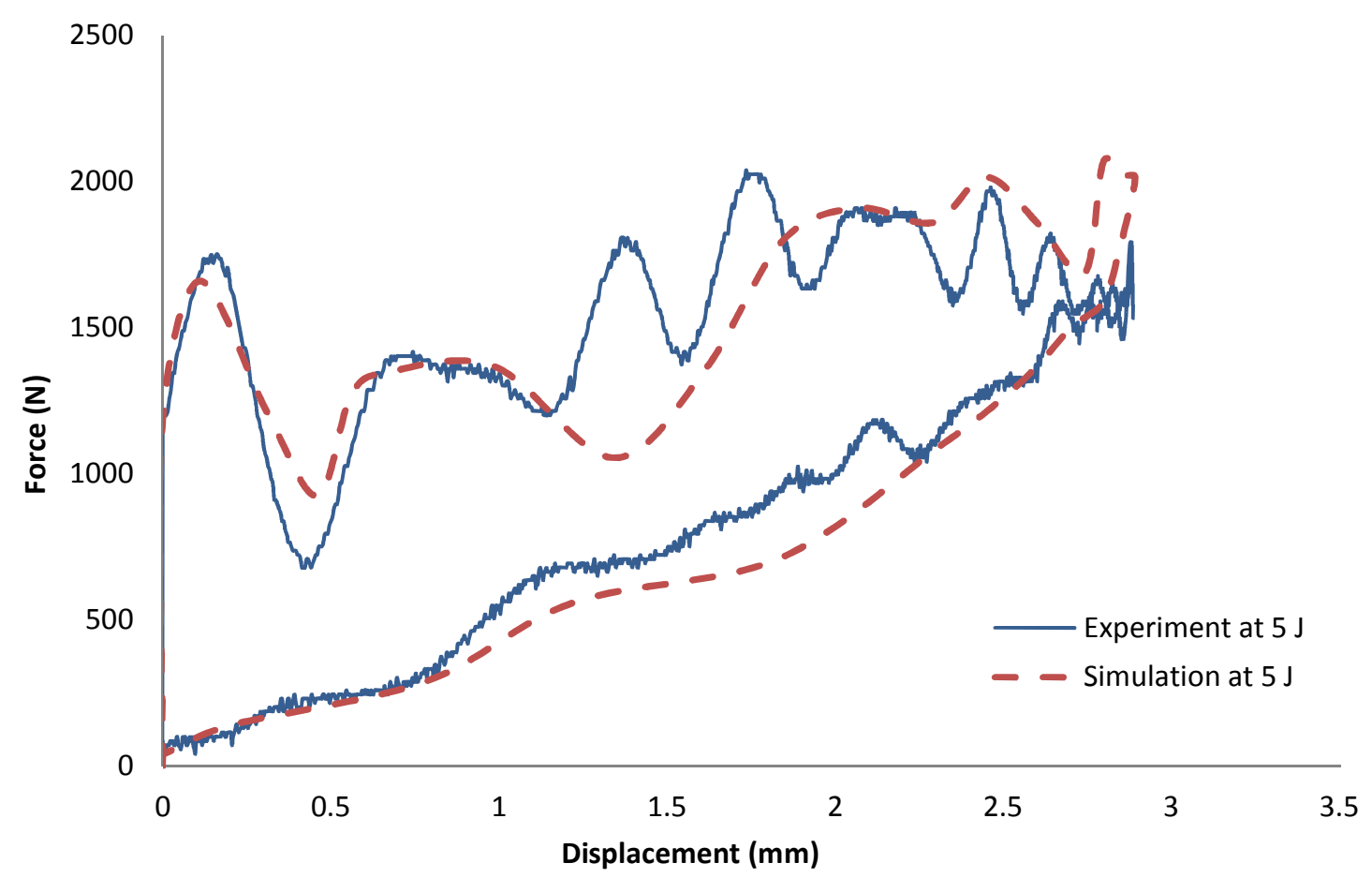

Figure 4: Force vs. displacement result and comparison between simulation and experiment of a $5 \mathrm{~J}$ impact

Falling weight impact tests were conducted by adjusting the drop heights of the $10 \mathrm{~mm}$ hemi-spherical steel impactor. Figure 4 shows the recorded force versus displacement from experiment observation and finite element analysis (FEA) prediction. A large oscillations zone is observed, which is an indication of material damage or rearrangement. The graph shows closed loops in which the area under the curve is the deformation energy that is initially transferred from the impactor to the oil pan surface and then given back from the oil pan surface to the impactor. The area included inside the loop refers to the energy absorbed during the impact. The maximum deflection for these tests was around $3 \mathrm{~mm}$.

For the low velocity projectiles impact, a search and selection of random granite stones were collected from regular roads in order to quantify the size and mass of stones likely to cause damage to the oil pan (Figure 5a). It was found that the average size profile susceptible to impact the oil pan base wall was 10 $\mathrm{mm}$ and the average mass was $22 \mathrm{~g}$. This assessment of road stones characteristics led to selection and design of specific projectile and impactor representing the average road stone (Figure $5 b$ and Figure $5 c$ ). The projectiles used our study are therefore made of aluminum as it has nearly the same density as granite gravels, $2700 \mathrm{~kg} / \mathrm{m}^{3}$. The projectiles were simulated as rigid material. The shape of typical projectile is equivalent to a stone with a triangular shape allowing the pointed end to hit the structure and it is also designed to permit a stable flying trajectory. 

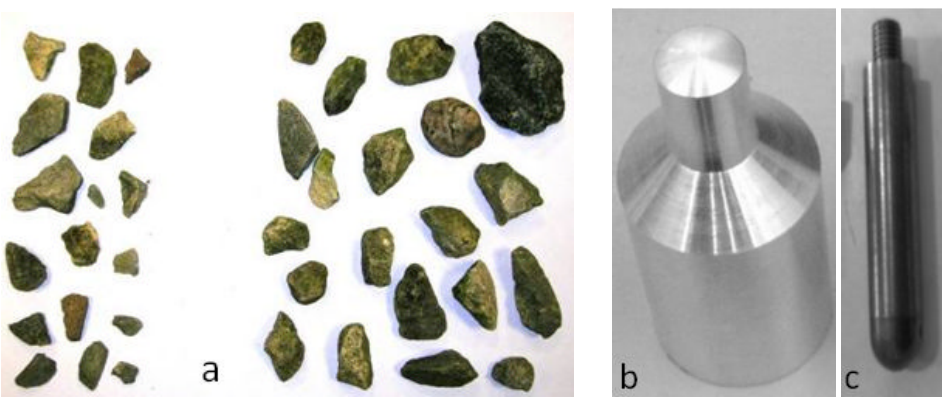

Figure 5: Prospection of stones (a) and typical flying projectile (b) and an example of falling weight impactor (c)

The structural geometry determines the target impact response [20]. The predictable analyses were necessarily based on the experimental testing in order to validate the numerical models. A lot of simulated impacts were realized all around the oil pan model and then compared to the experiments. A correlation was done by comparing experiments and simulated impact consequences made with the same conditions onto the oil pan. As an example, the following $5 \mathrm{~J}$ impacts numbered 1, 2 and 3 shown in Figure 6 were performed both by experiment and simulations. It can be observed that simulation renderings closely match the physical tests.

From Figure 6, impact $1(a, b)$ is straight into a rib at 90 degrees and shattered a third of ribs height. The base wall structure remained undamaged. In the simulation, the damage on the rib was slightly exaggerated, shattering half of its height. Impact $2(a, b)$ showed no dramatic damage, the impact touched the edge of a rib at 90 degrees and the projectile rebounded off the base wall structure creating a scratch on the rib. In the simulation, the scratch is noticed by the curvature created on the edge of the rib. Again, this was somewhat intensified then experimental observation. The experiment test showed a small dent on the edge of the rib but also a superficial scratch on the base wall structure. The oil pan model has a longer elastic behavior than expected material performance. The plastic strain of the model also increases too quickly once the yield stress is reached. Impact $3(a, b)$ involved two parallel ribs hit at 90 degrees where the $10 \mathrm{~mm}$ projectile cannot fit between them. The comparison between each test led to the same observations as the two previous impacts. The two ribs were damaged in both cases but given the prominent elastic characteristic of the model, the deformation in the model was amplified. The deflection of the base wall was well observed. However, the behavior of the ribbing was slightly accented and is less rigid than expected. 
Simulations
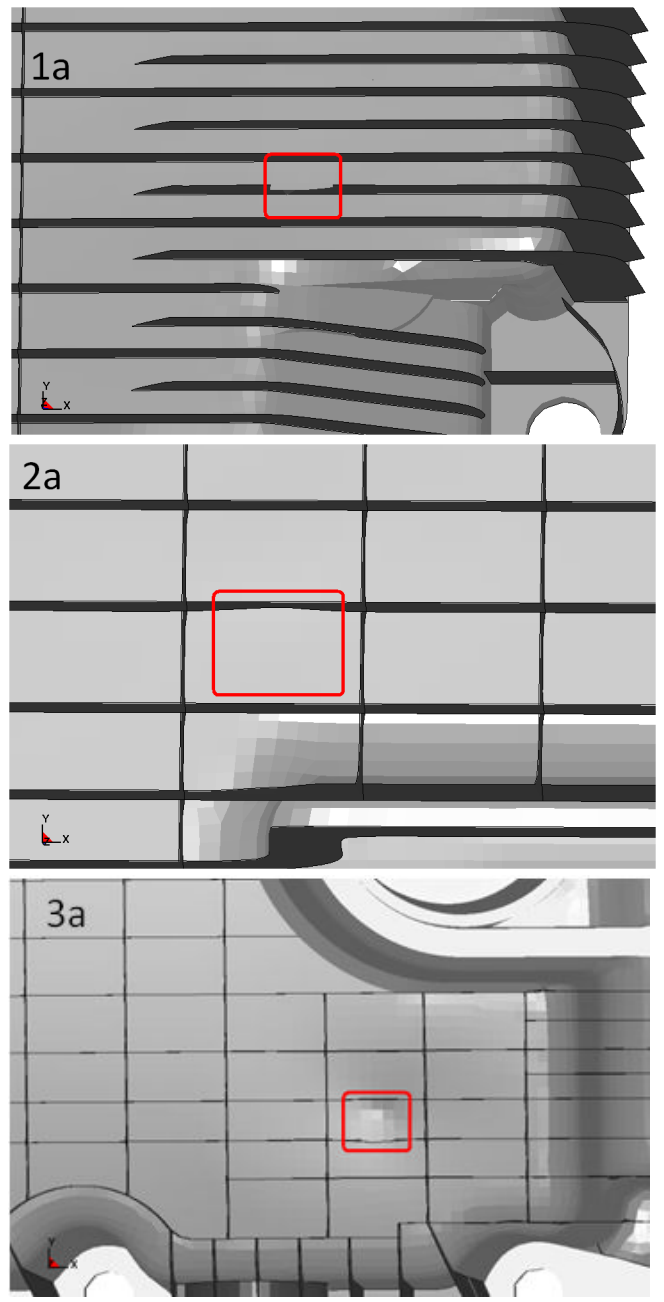

Experiments
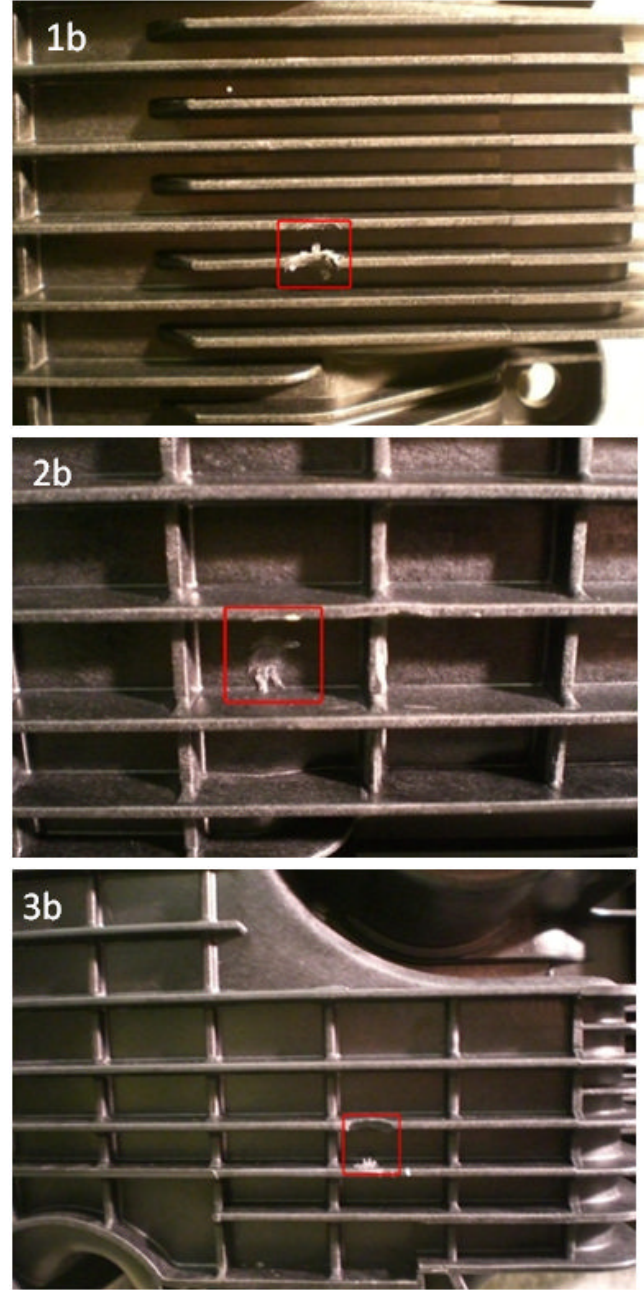

Figure 6: Results and comparison between simulations (left) and experiments (right) of three $5 \mathrm{~J}$ impacts

Figure 7 shows a $10 \mathrm{~mm}$ projectile impact sequence with the shock waves stress distribution propagating in circle away from the impact spot. When a shock wave reaches the edge it creates an accumulation of stress. Consequently, the ribbing stiffens the structure and therefore gives a dimensional stability to the oil pan. This acts like a shield against most of potential impacts by distributing the stress load all along the rib and on a much bigger surface area reducing the risk of base wall damage. 


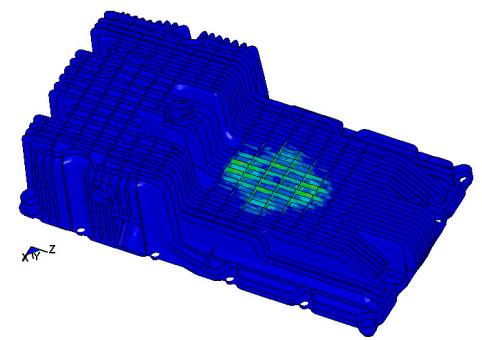

$\mathrm{T} 1$

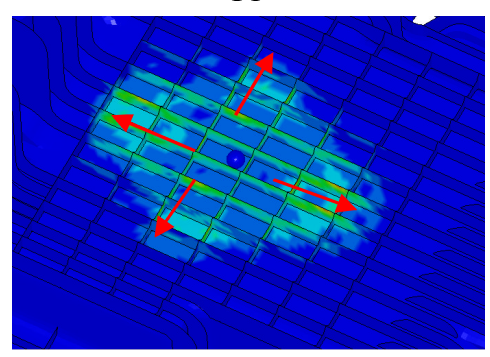

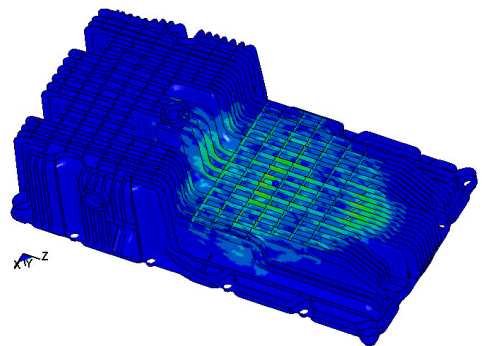

$\mathrm{T} 2$

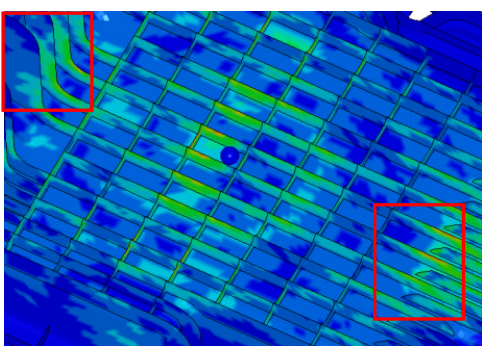

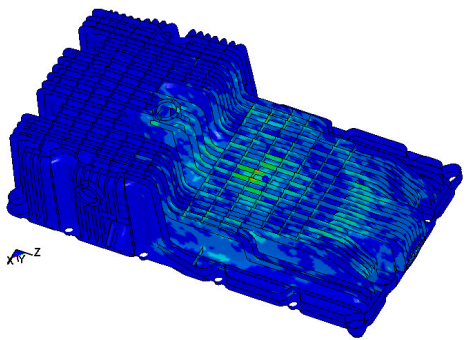

$\mathrm{T} 3$

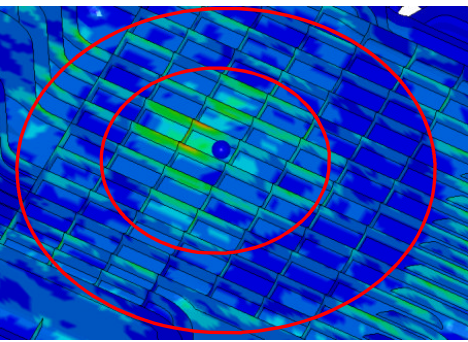

Figure 7: Snapshots of visual contours of effective stress of the oil pan impacted with a $10 \mathrm{~mm}$ sphere projectile at $14 \mathrm{~m} / \mathrm{s}$ (outer view)

For a first approach, the simulation is capable and further refinements in the material model are contemplated like for instance studying the strain rate dependency or also improving the stress-strain representation in the model.

The response to an impact loading and the way it dissipates the incident kinetic energy of flying road debris is far different to that of metals. For low and intermediate incident energies, metals absorb energy through elastic and plastic deformation. Although the latter may cause some permanent structural deformation, its consequences on the load-carrying capability of the component are usually small and its effects can generally be predicted using fracture mechanics principles. In composites however, the ability to undergo plastic deformation is extremely limited with the result that energy is frequently absorbed in creating large areas of fracture with ensuing reductions in both strength and stiffness [18]. Furthermore, the prediction of the post-impact load-bearing capability of a damaged composite structure is more difficult than for metals since the damage zone is generally complex in nature and consequently very difficult to characterize.

\section{Concluding remarks}

Glass filled polyamides have improved mechanical properties. The overall stiffness of an oil pan is improved by the flow property of the resin that enables long flow distances, thus enabling reliable 
molding of thin-walled sections. The stiffness and strength of the component are also increased by adding ribs at the edge of the pan. The experimental and FE results correlate closely in terms of material failure whilst tested with the same condition of load or impact. On the other hand, a change of a single factor like the impact velocity or the temperature will require a constant update of the stress-strain relation as the model is dependent on this relation and does not accommodate those variations. Due to material complexity, an impact on a given target when its intensity is very close to the material failure limit has variable outcome. In that case, predicted impact consequences are to be considered with an error margin where the component could pass or fail the impact and that is also true in the experiments.

\section{Acknowledgments}

The authors gratefully acknowledge the financial support from both the EPSRC grant CASE/CAN/07/40 and Research Council UK. The authors thank EATON Corp. UK for financial support on current research program at Cranfield University whose results are reported in this work. The authors also wish to acknowledge SABRE BALLISTICS for their support on gas gun testing and useful discussions. 


\section{References}

1. Zhao, G.P.; Cho, C.D. Damage initiation and propagation in composite shells subjected to impact. Compos. Struct. 2007, 78, 91-100.

2. Sınmazçelik, T.; Arıcı, A.A.; Günay, V. Impact-fatigue behaviour of unidirectional carbon fibre reinforced polyetheremide (PEI) composites. J. Mater. Sci. 2006, 41, 6237-6244.

3. Bartus, S.D.; Vaidya, U.K. Performance of long fiber reinforced thermoplastics subjected to transverse intermediate velocity blunt object impact. Compos. Struct. 2005, 67, 263-277.

4. Dear, J.P.; Lee, H.; Brown, S.A. Impact damage processes in composite sheet and sandwich honeycomb materials. Int. J. Impact Eng. 2005, 32, 130-154.

5. Santulli, C.; Brooks, R.; Long, A.C.; Warrior, N.A.; Rudd, C.D. Impact properties of compression moulded commingled E-glass-polypropylene composites. Plast. Rubber Compos. Process. Appl. 2002, 31, 270-277.

6. Putnoki, I.; Moos, E.; Karger-Kocsis, J. Mechanical performance of stretched knitted fabric glass fibre reinforced poly(ethylene terephthalate) composites produced from commingled yarn. Plast. Rubber Compos. Process. Appl. 1999, 28, 40-46.

7. Bigg, D.M. The impact behavior of thermoplastic sheet composites. J. Reinf. Plast. Compos. 1994, 13, 339-354.

8. Cantwell, W.J.; Morton, J. The impact resistance of composite materials - a review. Composites 1991, 22, 347-362.

9. Naik, N.K.; Meduri, S. Polymer matrix composites subjected to low velocity impact: effect of laminate configuration, Compos. Sci. Technol. 2001, 61, 1429-1436.

10. Alcock, B.; Cabrera, N.O.; Barkoula, N.M.; Wan, Z.; Peijs, T. The effect of temperature and strain rate on the impact performance of recyclable all-polypropylene composites. Composites B 2007, 67, 2061-2070.

11. Alcock, B.; Cabrera, N.O.; Barkoula, N.M.; Peijs, T. Low velocity impact performance of recyclable all-polypropylene composites. Compos. Sci. Technol. 2006, 66, 1724-1737.

12. $\mathrm{Xu}, \mathrm{T}$.; Farris, R.J. Comparative studies of ultra high molecular weight polyethylene fiber reinforced composites. Polym. Eng. Sci. 2007, 4, 1544-1553.

13. Walsh, S.M.; Scott, B.R.; Spagnuolo, D.M. The development of a hybrid thermoplastic ballistic material with application to helmets. Technical Report ARL-TR-3700. U.S. Army Research Laboratory, Aberdeen Proving Ground, USA, 2005.

14. Reyes, G.; Sharma, U. Modeling and damage repair of woven thermoplastic composites subjected to low velocity impact. Compos. Struct. 2010, 92, 523-531.

15. Bartus, S.D.; Vaidya, U.K. Performance of long fiber reinforced thermoplastics subjected to transverse intermediate velocity blunt object impact. Compos. Struct. 2005, 67, 263-277.

16. Duan, Y., Saigal, A., Greif, R. Impact behavior and modeling of engineering polymers. Polym. Eng. Sci. 2003, 43, 112-114.

17. Viana, J.C., Cunha, A.M., Billon, N. Experimental characterization and computational simulations of the impact behavior of injection-molded polymers. Polym. Eng. Sci., 2007, 47, 337-346. 
18. Lobo, H., Hurtado, J. Characterization and modeling of nonlinear behavior of plastics. ABAQUS User Conference. Boston, 2006.

19. Lobo, H. Methodology for selection of material models for plastics impact simulation. $10^{\text {th }}$ International LS-Dyna User's Conference. Detroit, 2006.

20. Mouti, Z., Westwood, K., Kayvantash, K., Njuguna, J. Low velocity impact behavior of glass filledreinforced thermoplastic engine components. Materials 2010, 3, 2463-2473. 